\title{
DIFICULTADES Y CONTRADICCIONES \\ EN LA RESPUESTA EDUCATIVA PARA LOS ALUMNOS CON NECESIDAD ESPECÍFICA DE APOYO EDUCATIVO (ACNEAE) ${ }^{\mathrm{I}}$
}

\section{Difficulties and contradictions in the educational response for students with specific need for educational support (ACNEAEs)}

\author{
Roberto RuIz ANDRÉs \\ Universidad de Burgos \\ Correo-e: rrandres@ubu.es \\ Recibido: 2I de febrero de 2020 \\ Envío a informantes: 4 de marzo de 2020 \\ Aceptación definitiva: 27 de abril de 2020
}

Las escuelas discriminan a los estudiantes, y los mecanismos que provocan esta discriminación tienen que ver, de forma muy significativa, con la concepción de la diversidad presente en los centros escolares (Aguado y Ballesteros, 2012: 13. Citado por Ruiz Andrés, 2016: 156).

Resumen: Se considera que la respuesta educativa que se viene ofreciendo al alumnado con necesidad específica de apoyo educativo en institutos o centros de educación secundaria es insuficiente y claramente mejorable. Tras poner de manifiesto que en dichos centros se vulnera el principio de atención a la diversidad que debe impregnar toda la enseñanza básica, así como el derecho a la plena participación, aprendizaje y éxito de todos que proclama el modelo de escuela inclusiva, en el presente documento se reflexiona sobre cómo la normativa actual clasifica al alumnado que requiere una atención educativa diferente a la ordinaria, estableciendo una marcada barrera conceptual

\footnotetext{
Nos ceñiremos al contexto de la Comunidad de Castilla y León y la etapa de Educación Secundaria Obligatoria; no obstante, seguramente, muchas de las ideas y argumentaciones aquí presentadas puedan ser válidas además para la etapa de Educación Primaria y generalizarse también al resto de las Comunidades Autónomas de España.
} 


\section{DIFICULTADES Y CONTRADICCIONES EN LA RESPUESTA EDUCATIVA \\ I 50 PARA LOS ALUMNOS CON NECESIDAD ESPECÍFICA DE APOYO EDUCATIVO (ACNEAE) ROBERTO RUIZ ANDRÉS}

entre los alumnos con necesidades educativas especiales (ACNEE) y el resto de los grupos de alumnos con necesidad específica de apoyo educativo (ACNEAE) que también pueden presentar un desfase significativo de dos o más cursos en el nivel de competencia curricular; mientras que para los primeros se reservan exclusivamente las adaptaciones curriculares significativas (ACS), la respuesta educativa que se prevé para los segundos (ACNEAE que no son ACNEE) queda en la más absoluta indefinición, cayendo además en graves incongruencias a la hora de diseñar y desarrollar una propuesta educativa adecuada y, sobre todo, a la hora de abordar una de las partes esenciales del proceso de enseñanza/aprendizaje: la evaluación. En este sentido, sin renunciar por ello a reclamar una trasformación más profunda y estructural del Sistema Educativo, se propone un cambio en la normativa para que en las materias en las que este alumnado recibe apoyo específico, normalmente Lengua y Matemáticas, la evaluación tome como referentes los elementos planteados en las propuestas curriculares individuales que se diseñan a partir de su nivel de competencia curricular.

Palabras clave: respuesta educativa; alumno con necesidad específica de apoyo educativo; necesidades educativas especiales; adaptación curricular significativa.

Aвstract: The educational response that is being offered to students with a specific need for educational support in secondary schools or centers is considered insufficient and clearly improvable. After showing that, in these centers, the principle of attention to diversity that must permeate all basic education is violated, as well as the right to full participation, learning and success of all that the inclusive school model proclaims, in this document reflects on how the current regulations classify students who require different educational attention than ordinary ones, establishing a marked conceptual barrier between students with special educational needs (ACNEES) and the rest of the groups of students with specific need for educational support (ACNEAEs) that may also present a significant gap of two or more courses at the level of curricular competence; while significant curricular adaptations (ACS) are reserved exclusively for the former, the educational response that is foreseen for the latter (ACNEAES that are not ACNEES), remains in the absolute absence of definition, also falling into serious inconsistencies when designing and develop an appropriate educational proposal and, above all, when addressing one of the essential parts of the teaching / learning process: evaluation. In this sense, without renouncing for this reason to demand a more profound and structural transformation of the Educational System, a change in the regulations is proposed so that in the subjects in which this student receives specific support, normally Language and Mathematics, the evaluation takes as reference the proposed elements in the individual curricular proposals that are designed based on their level of curricular competence.

KEY WORDS: educational response; student with specific need for educational support; special educational needs; meaningful curricular adaptation.

\section{Introducción}

\footnotetext{
A

PARTIR DE LA OBSERVACIÓN Y REFLEXIÓN sobre la propia práctica como maestro de Pedagogía Terapéutica en un instituto de educación secundaria, venimos constatando que la respuesta educativa que se está ofreciendo en los centros educativos de educación secundaria a determinados alumnos con necesidad específica de apoyo educativo (en adelante ACNEAE) está lejos de ser una respuesta propia de una educación inclusiva y de calidad.
} 
DIFICULTADES Y CONTRADICCIONES EN LA RESPUESTA EDUCATIVA PARA LOS ALUMNOS CON NECESIDAD ESPECÍFICA DE APOYO EDUCATIVO (ACNEAE) ROBERTO RUIZ ANDRÉS

Articular la adecuada respuesta a la diversidad de intereses, necesidades, motivaciones, capacidades, estilos y ritmos de aprendizaje, entre otros, del alumnado desde un currículo común y comprensivo siempre ha generado, en la práctica, controversias, contradicciones, dudas e incertidumbres que aún hoy persisten después de que hayan trascurrido ya casi 30 años desde que se hicieran esos planteamientos en nuestro sistema educativo. Así lo expresábamos en Ruiz Andrés (2016): «La respuesta educativa a la diversidad [...] pasa por la necesaria y difícil tarea de articular la educación común y básica, con la diversidad de intereses, capacidades, necesidades, ritmos y estilos de aprendizaje, etc. Éste ha sido, y es aún hoy, el dilema que desde i99o se planteó la escuela española y que, en realidad, está sin resolver» (p. 278). Con esas dificultades venimos desde entonces desarrollando nuestra labor docente observando que ni las normativas (nacionales y autonómicas), ni las prácticas y dinámicas implementadas en los centros educativos en relación a la atención a la diversidad hayan contribuido eficazmente a resolver tal dilema ni a avanzar hacia una respuesta educativa adecuada y de calidad para muchos alumnos que requieren una atención educativa diferente a la ordinaria, los ACNEAE, que les permita acceder y/o cursar el currículo común y alcanzar los objetivos educativos establecidos para todos los alumnos.

La investigación y la literatura sobre el tema señalan múltiples factores como causantes de que, para muchos alumnos, esa respuesta educativa no esté siendo la más adecuada. Entre ellos destacamos algunos de los directamente relacionados con la propia escuela: un currículo hegemónico, academicista, memorístico, verbalista descontextualizado que constituye una «barrera que dificulta las dinámicas de pertenencia y participación en la vida escolar de determinados alumnos, así como un impedimento para la igualdad de oportunidades» (Echeita, 2008: II); además, un concepto de la evaluación que desvirtúa la esencia misma de esta y la convierte en un instrumento de mera calificación y descalificación que deriva en exclusión para muchos alumnos; así mismo, una metodología obsoleta, centrada en la mera trasmisión de contenidos del libro de texto; también, muchas de las prácticas generalizadas en los años 90 derivadas de la implementación del modelo de la integración educativa como la misma evaluación psicopedagógica, la clasificación del alumnado en categorías diagnósticas, la organización de los apoyos educativos sacando del aula ordinaria a determinados alumnos, entre otras (Echeita y Calderón, 20I4). Con respecto a esta última causa, hemos de aclarar que el modelo de necesidades educativas supuso un gran avance respecto a la consideración de la diversidad y de la respuesta educativa adecuada a la misma, acarreando consigo un aumento de recursos, implementación de dinámicas y prácticas de integración que fueron de gran utilidad en su momento; sin embargo, esas mismas dinámicas y prácticas tan arraigadas en el Sistema son las que están hoy frenando fuertemente el avance hacia un modelo de escuela inclusiva que garantice la atención a la diversidad desde un currículo común mediante la participación y el éxito de todos los alumnos en los centros de educación secundaria.

No es el propósito del presente documento entrar en el análisis de estos factores (un análisis detallado podemos encontrarlo en Ruiz Andrés [20I6]), lo que pretendemos es destacar un factor más relacionado con esa respuesta educativa a determinados alumnos que viene siendo mediocre y claramente mejorable. La idea central de la discusión que se plantea en el presente artículo tiene su origen en la clasificación que la normativa actual, tanto de ámbito nacional (LOE, 2006-LOMCE, 2OI3) como autonómico en Castilla y León (Resolución de 17 de agosto de 2009, Orden EDU/II52/20Io, 


\section{DIFICULTADES Y CONTRADICCIONES EN LA RESPUESTA EDUCATIVA I 2 PARA LOS ALUMNOS CON NECESIDAD ESPECÍFICA DE APOYO EDUCATIVO (ACNEAE) ROBERTO RUIZ ANDRÉS}

Instrucción de 24 de agosto de 20I7), realiza de este alumnado que requiere una atención educativa diferente a la ordinaria, estableciendo una marcada barrera conceptual entre los alumnos con necesidades educativas especiales (ACNEE) y el resto de los grupos de alumnos con necesidad específica de apoyo educativo (ACNEAE) que también pueden presentar, y de hecho presentan, un desfase significativo de dos o más cursos en el nivel de competencia curricular. Así, de esta clasificación se derivan determinadas consecuencias prácticas: mientras que para los primeros (ACNEE) se reservan exclusivamente las adaptaciones curriculares significativas (ACs) que toman como referentes para la evaluación los elementos del currículo establecidos en ellas, la respuesta educativa que se prevé para los segundos (ACNEAE que no son ACNEE) queda en la más absoluta indefinición, cayendo además en graves incongruencias a la hora de abordar, en la práctica, una de las partes esenciales del proceso de enseñanza/ aprendizaje: la evaluación.

En este sentido, de modo más concreto, observamos que, en la mayoría de los institutos o centros de educación secundaria que conocemos, la respuesta educativa para este alumnado al que nos referimos (ACNEAE que no son ACNEE) se articula frecuentemente de la siguiente manera: a) Para las materias de Lengua y Matemáticas se elaboran propuestas educativas individuales las cuales se desarrollan normalmente (total o parcialmente) en las aulas de apoyo (aulas de pedagogía terapéutica y aulas de educación compensatoria). Estas programaciones individuales, llamadas Planes de Apoyo Individual o Planes de Intervención Individual, son diseñadas por los profesionales de pedagogía terapéutica y de educación compensatoria a partir del nivel de competencia curricular (NCC) de cada alumno, con frecuencia dos cursos por debajo con respecto al nivel de su grupo-clase; sin embargo, en el momento de evaluar se deben tomar (según la normativa) los referentes ordinarios fijados en la Programación de Aula. Así, este alumnado está abocado, ya a priori, a calificaciones negativas en estas asignaturas.

b) Respecto al resto de las materias, este alumnado las cursa en las aulas ordinarias con algunas adaptaciones metodológicas que, en el mejor de los casos, determinados profesores aplican siguiendo algunas orientaciones proporcionadas por el Departamento de Orientación. Tampoco en estas materias suelen obtener resultados satisfactorios en la evaluación.

Como consecuencia de estos planteamientos de respuesta educativa, el resultado final al que se llega es que un gran número de alumnos con necesidad específica de apoyo educativo que pertenece a los grupos Alumnos con Necesidad de Compensación Educativa (ANCE) -cualquier tipología-y Dificultad Específica de Aprendizaje y/o Bajo Rendimiento Académico -en las tipologías de: Lectura, Escritura, Matemáticas, Lectoescritura, Capacidad Intelectual Limite... (Instrucción 24 de agosto de 2017 -Castilla y León-) están abocados al fracaso escolar generalizado, a la exclusión y al abandono escolar, no llegando la mayoría de ellos a titular en ESO. La conclusión es que la respuesta educativa para un número importante de alumnos es claramente insuficiente e inadecuada debido, en parte, a incongruencias en la propia normativa que la regula. De este modo, entendemos que se vulnera gravemente el principio fundamental de atención a la diversidad que nuestro sistema establece para la educación básica, así como el derecho a la plena participación, aprendizaje y éxito de todos que promueve el modelo de escuela inclusiva. 


\section{DIFICULTADES Y CONTRADICCIONES EN LA RESPUESTA EDUCATIVA PARA LOS ALUMNOS CON NECESIDAD ESPECÍ́FICA DE APOYO EDUCATIVO (ACNEAE) ROBERTO RUIZ ANDRÉS \\ I. La atención a la diversidad como principio fundamental que debe regir la enseñanza básica. La educación inclusiva}

El hecho de que en el presente documento abordemos cuestiones muy concretas, vinculadas a la práctica educativa imperante, tan apartada a veces de los postulados y premisas que se establecen bajo el modelo de la inclusión educativa, no significa que renunciemos a las demandas y aspiraciones que en dicho paradigma se proclaman; más bien al contrario, en este primer apartado pretendemos apoyarnos en lo que debiera ser para poner de manifiesto cuán lejos estamos de ello a causa de esa «falta de voluntad y determinación» a la que apuntan Echeita y Domínguez Gutiérrez (20II), [...] «por qué hacemos lo que hacemos y por qué no hacemos lo que decimos que deberíamos hacer» (p. 25).

La normativa educativa actual (LOE, 2006; LOMCE, 2013) establece la atención a la diversidad como principio fundamental con repercusiones pedagógicas y organizativas:

La atención a la diversidad se establece como principio fundamental que debe regir toda la enseñanza básica, con el objetivo de proporcionar a todo el alumnado una educación adecuada a sus características y necesidades (Preámbulo, LOE, 2006).

Sin perjuicio de que a lo largo de la enseñanza básica se garantice una educación común para los alumnos, se adoptará la atención a la diversidad como principio fundamental. Cuando tal diversidad lo requiera, se adoptarán las medidas organizativas y curriculares pertinentes, según lo dispuesto en la presente Ley (LOE, 2006: art. 4).

Así lo expresábamos en un trabajo anterior (Ruiz Andrés, 2016):

[...] en nuestro sistema educativo se postula que la atención a la diversidad debe ser entendida como el conjunto de actuaciones educativas dirigidas a dar respuesta a las diferentes capacidades, ritmos y estilos de aprendizaje, motivaciones e intereses, situaciones sociales, culturales, lingüísticas y de salud del alumnado. Constituye, por tanto, un principio fundamental que debe regir toda la enseñanza básica cuya finalidad es asegurar la igualdad de oportunidades de todos los alumnos ante la educación y evitar, en la medida de lo posible, el fracaso escolar y el consecuente riesgo de abandono del sistema educativo, de tal modo que todos puedan alcanzar las capacidades y competencias básicas para participar activamente en la sociedad (p. 174).

En dicho trabajo poníamos en relieve que los centros educativos, especialmente los de educación secundaria, no suelen hacer de este principio de atención a la diversidad el eje principal de su actividad educativa, al menos no con la intensidad deseada. Frecuentemente este principio queda olvidado o relegado a segundo plano, cuando por Ley cada centro debe tener definido su Proyecto Educativo que «[...] recogerá la forma de atención a la diversidad del alumnado [...] y deberá respetar el principio de no discriminación y de inclusión educativa como valores fundamentales» (LOMCE, 2013: art. I2I). Sin embargo, observamos como en la vida de muchos centros de educación secundaria esta atención a la diversidad se concreta frecuentemente en prácticas de exclusión como sacar al alumnado a aulas de apoyo (Pedagogía Terapéutica y Educación Compensatoria) y queda reducida, en el mejor de los casos, a actuaciones y actividades inclusivas anecdóticas y aisladas, no llegando a impregnar realmente las acciones educativas cotidianas del centro, ni los procesos de enseñanza-aprendizaje, ni los procesos de evaluación que tienen lugar en el centro y en las aulas ordinarias. 


\section{DIFICULTADES Y CONTRADICCIONES EN LA RESPUESTA EDUCATIVA I 54 PARA LOS ALUMNOS CON NECESIDAD ESPECÍFICA DE APOYO EDUCATIVO (ACNEAE) ROBERTO RUIZ ANDRÉS}

Por lo tanto, apelamos a la responsabilidad de la Administración Educativa, así como de los profesionales de la educación, para no permitir que se vulnere, en modo o grado alguno, este principio de atención a la diversidad establecido (por Ley) como fundamental; además, apelamos a la necesidad de hacerlo plenamente efectivo en la vida cotidiana de los centros educativos.

Por otro lado, la escuela de hoy debe estar presidida necesaria y obligatoriamente por el paradigma de la inclusión educativa. La educación inclusiva se concibe como un derecho de todos a una educación de calidad reconocido por Convenciones y Declaraciones Internacionales (Declaración Mundial sobre Educación para Todos -Unesco, 1990-; Declaración de Salamanca, I994; Conferencia de Ginebra de 2008...). «Un derecho esencial que ha de garantizarse a todas las personas, sin ningún género de discriminación o exclusión, es un valor y un principio fundamental, abiertamente ideológico, no fáctico» (Escudero y Martínez, 20II: 88. Citado por Echeita y Domínguez Gutiérrez, 20II: 26). Derecho y justicia son los fundamentos que sustentan la educación inclusiva.

Por otra parte, las normativas de ámbito nacional deben impulsar con determinación las culturas, políticas y prácticas que hagan efectivo ese derecho. El principio de atención a la diversidad debe, como es prescriptivo, estar presidido por el de equidad y por el de inclusión. La LOE (2006) - LOMCE (2013) establece como principio: «La equidad, que garantice la igualdad de oportunidades para el pleno desarrollo de la personalidad a través de la educación, la inclusión educativa, la igualdad de derechos y oportunidades que ayuden a superar cualquier discriminación...» (art. I). Con toda claridad se establece en el Título II, sobre Equidad en la educación: "La atención integral al alumnado con necesidad específica de apoyo educativo se iniciará desde el mismo momento en que dicha necesidad sea identificada y se regirá por los principios de normalización e inclusión» (LOMCE, 2013: art. 7I). En este sentido, la equidad, unida siempre a la idea de calidad en educación y de igualdad de oportunidades, a menudo se olvida, se tergiversa y se confunde en la práctica. Compartimos con Bolívar la idea de equidad:

En definitiva, la equidad en educación implica cuidar que las desigualdades no condicionen el aprendizaje y el rendimiento poniendo los medios y recursos compensatorios en alumnos desfavorecidos, persiguiendo la igualdad de oportunidades, la igualdad de enseñanza de calidad para todos, igualdad de conocimiento y éxito escolar e igualdad de resultados (Bolívar, 2005. Citado por Ruiz Andrés, 2016: 153).

A nivel autonómico, en la Comunidad de Castilla y León se ha establecido el II Plan de Atención a la Diversidad para el periodo 20I7-2022, «con el propósito de avanzar hacia un paradigma educativo que es el de la inclusión educativa». Dicho Plan está articulado en 6 líneas estratégicas con objetivos específicos dentro de cada una [...] «basados en principios de equidad, inclusión, normalización, proximidad, accesibilidad universal y diseño para todos, participación, eficiencia y eficacia, sensibilización, coordinación y prevención» (Preámbulo). Esperamos que estas declaraciones realizadas por la Administración Autonómica se materialicen en hechos concretos en la vida de los centros educativos y en la de las personas que en ellos se educan:

[...] apostaremos decididamente por un modelo de «Inclusión Educativa» que responda a las demandas de la sociedad actual, facilitando la igualdad de oportunidades, y evitando, 


\section{DIFICULTADES Y CONTRADICCIONES EN LA RESPUESTA EDUCATIVA PARA LOS ALUMNOS CON NECESIDAD ESPECÍFICA DE APOYO EDUCATIVO (ACNEAE) ROBERTO RUIZ ANDRÉS}

con una educación de calidad, la generación de sectores de población en riesgo de exclusión social (p. 4).

Este II Plan que presentamos debe entenderse como una referencia en materia de atención a la diversidad en Castilla y León y pretende establecer las líneas estratégicas de actuación en nuestra Comunidad Autónoma desde una visión inclusiva de la educación. Es resultado del compromiso de la Administración Educativa actuar desde su esfera de responsabilidad, tomando las medidas necesarias para alcanzar los objetivos de la educación para todas y todos, dando así un paso más hacia la construcción de una educación equitativa y de alta calidad (p. 7).

Además de las normativas, también se cuenta con la investigación y la literatura como elementos de impulso hacia la inclusión educativa. Afirman Echeita y Domínguez Gutiérrez (20II) que «siendo una empresa de enorme complejidad y calado, no estamos faltos de conocimientos y capacidades para llevarla a cabo» (p. 25). La literatura sobre el tema viene analizando la naturaleza, el concepto y significado de la educación inclusiva y definiendo las premisas esenciales de la misma entre las que destacamos: la diversidad es un valor que enriquece los procesos de enseñanza-aprendizaje. No es solamente estar, sino pertenecer, aprender y participar juntos, y éxito para todos (Booth y Ainscow, 2002; Arnáiz, 2003; Ainscow y Miles, 2008). Por la importancia que tiene para las cuestiones de las que nos ocuparemos después, destacamos esta idea de la participación:

La participación en educación implica ir más allá que el acceso. Implica aprender con otros y colaborar con ellos en el transcurso de las clases y las lecciones. Supone una implicación activa con lo que se está aprendiendo y enseñando y cabría decir lo mismo con relación a la educación que se está experimentado. Pero la participación también implica ser reconocido por lo que uno es y ser aceptado por esto mismo (Booth, 2002: 2. Citado por Echeita y Domínguez Gutiérrez: 28).

Entonces, si se cuenta con el impulso de declaraciones y convenciones internacionales, con un marco normativo de ámbito nacional y autonómico y con el conocimiento suficiente que aporta la investigación, nos volvemos a preguntar, con Echeita y Domínguez Gutiérrez (20II), [...] «por qué hacemos lo que hacemos y por qué no hacemos lo que decimos que deberíamos hacer» (p. 25). Por qué estamos realmente tan alejados de que el principio de atención a la diversidad y las premisas del modelo de inclusión tengan en la práctica y en la vida cotidiana de los centros de educación secundaria el calado y la importancia que les corresponde. No decimos que resulte fácil. La educación inclusiva tiene carácter complejo, poliédrico y polisistémico, paradójico y contradictorio: la inclusión se refiere a todos los alumnos, pero al mismo tiempo debe preocuparse con mayor intensidad por los más vulnerables; si se diseñan medidas concretas de atención a la diversidad y prácticas de discriminación positiva dirigidas a los alumnos más vulnerables, estas mismas medidas, por un lado, estigmatizan por la valoración social negativa que tienen y por la interiorización que hacen de ellas y, por otro lado, resulta paradójico que los usuarios pongan en valor estas mismas medidas específicas y las reclamen (Echeita, 2008; Echeita y Domínguez Gutiérrez, 20II). Además, como apuntábamos en la introducción, existen dificultades para articular un currículo común y comprensivo con la diversidad de capacidades, intereses, necesidades, etc., sumado a las múltiples causas que están impidiendo el abandono del 


\section{DIFICULTADES Y CONTRADICCIONES EN LA RESPUESTA EDUCATIVA I 56 PARA LOS ALUMNOS CON NECESIDAD ESPECÍfICA DE APOYO EDUCATIVO (ACNEAE) ROBERTO RUIZ ANDRÉS}

modelo de necesidades educativas especiales y el avance hacia el modelo inclusivo y de eliminación de barreras para la participación y el aprendizaje, entre ellas, multitud de prácticas ancladas aún en concepciones propias del modelo del déficit y en el modelo de necesidades educativas especiales. Como vemos, son muchos y muy diversos los obstáculos que pueden explicar ese distanciamiento entre el ser y el deber ser de la inclusión, sin embargo, apuntamos con Echeita y Domínguez Gutiérrez (20II) a la «falta de voluntad y determinación» para llevar a cabo en la práctica esta empresa y sus procesos.

A modo de ejemplo, formulamos a continuación unas cuestiones cuya respuesta a las mismas nos podría ayudar a tomar conciencia de dónde se sitúa realmente el centro educativo de educación secundaria en relación con el marco general que establece la normativa sobre atención a la diversidad: a) ¿En el centro se concibe la atención a la diversidad como un principio fundamental que impregna toda la actividad educativa? ¿Podemos enumerar las actuaciones pedagógicas y organizativas concretas que se están desarrollando en el centro y en cada aula que lo ponen de manifiesto? b) ¿Se concibe en la comunidad educativa (centro y familias) la diversidad como un valor que beneficia a todos y que enriquece los procesos de enseñanza-aprendizaje? ¿En qué actuaciones concretas o indicadores se observa? c) ¿Podemos afirmar con rotundidad que la participación, el aprendizaje y el éxito de todos es la preocupación fundamental de toda la comunidad educativa? d) ¿Tenemos identificadas las barreras que obstaculizan e impiden la participación, el aprendizaje y el éxito de todos los alumnos y qué actuaciones concretas se vienen desarrollando en el centro y en cada aula para reducirlas/eliminarlas? En suma, deberíamos preguntarnos si las concepciones, los modos organizativos y las actuaciones que se llevan a cabo en el centro y en las aulas están garantizando realmente el derecho de todos a una educación de calidad, con equidad, en la que no se excluye a nadie, en la que se acepta de manera plena la diversidad de todos los alumnos y en la que se ofrecen las mismas oportunidades educativas y de éxito para todos.

Sin renunciar por ello a reivindicar la tan necesaria como anhelada trasformación profunda del Sistema Educativo que reclaman tantos y tantos autores y que exige un enfoque sistémico para abordar una empresa, la de la inclusión y sus procesos, compleja, dilemática, contradictoria y paradójica (Echeita, 2008; Echeita y Domínguez Gutiérrez, 20II), vamos a ir concretando las cuestiones que más directamente se relacionan con los argumentos que queremos exponer.

\section{Alumnos con necesidad específica de apoyo educativo (ACNEAE) y alumnos con necesidades educativas especiales (ACNEE). Conceptualización}

Una vez que hemos puesto de manifiesto que el principio de atención a la diversidad en el marco de una escuela inclusiva no constituye, como debiera, el eje fundamental de la vida de los centros de educación secundaria, pasamos ahora a analizar la evolución de la conceptualización y clasificación que se ha venido realizando del alumnado que presenta dificultades para acceder y/o cursar el currículo común que se ofrece para todos. Consideramos que esta conceptualización tiene gran repercusión sobre la organización de la respuesta educativa y puede estar en el origen del tema central que planteamos en el presente artículo. 


\section{I. Alumnos con necesidades educativas especiales (ACNEE)}

La Reforma del Sistema Educativo impulsada por la LOGSE (I990) introdujo en el sistema educativo el concepto de necesidades educativas especiales (NEE) que aún hoy se maneja. Esto supuso una nueva reconceptualización de la educación especial basada, al menos, en dos ideas fundamentales. La primera es que los fines de la educación son los mismos para todos los alumnos, y todos, a través de un currículo común y comprensivo, deben aspirar a alcanzarlos. La segunda idea sobre la que se fundamenta esa reconceptualización es que se pone de manifiesto que las NEE pueden ser temporales o permanentes y tienen un carácter continuo, relativo e interactivo, es decir, que no van a depender solo del déficit que presente el propio alumno, sino que las necesidades dependerán de la ayuda y/o ajuste educativo que se le ofrezca. (Una exposición detallada la podemos encontrar en Ruiz Andrés, 20I6: I7I y siguientes).

El Ministerio de Educación y Ciencia, a través del Centro Nacional de Recursos para la Educación Especial, define quién es un alumno que presenta NEE:

Un alumno tiene necesidades educativas especiales cuando presenta dificultades mayores que el resto de los alumnos para acceder a los aprendizajes que se determinan en el currículo que le corresponde por su edad (bien por causas internas, por dificultades o carencias en el entorno sociofamiliar o por una historia de aprendizaje desajustada) y necesita, para compensar dichas dificultades, adaptaciones de acceso y/o adaptaciones curriculares significativas en varias áreas del currículo (CNREE, I992: 20).

En otro texto, el mismo Ministerio lo recoge así:

Decir que un alumno presenta necesidades educativas especiales es una forma de decir simplemente que, para el logro de los fines de la educación, no son suficientes las actuaciones habituales que su profesor desarrolla con la mayoría de los alumnos del grupo y que, por ello debe revisar su actuación y adecuarla a las necesidades particulares del alumno en cuestión (MEC, 1994).

De la misma forma, el Real Decreto 696/1995, de 28 de abril, sobre ordenación de la educación de los alumnos con necesidades educativas especiales, define claramente cómo pueden ser las NEE y a qué causas se asocian:

Es objeto del presente Real Decreto la regulación de las condiciones para la atención educativa a los alumnos con necesidades especiales, temporales o permanentes, asociadas a su historia educativa y escolar, o debidas a condiciones personales de sobredotación y de discapacidad psíquica, motora o sensorial (art. I).

Así pues, en los años 9o, se establece un solo grupo de alumnado que tiene mayores dificultades que el resto para acceder y cursar el currículo, los ACNEE. Esas NEE que presentan algunos alumnos pueden ser temporales o permanentes y estar asociadas a su historia educativa y escolar, debidas a condiciones personales de sobredotación y de discapacidad psíquica, motora o sensorial, es decir, a cualquier causa.

Hemos de adelantar aquí un hecho importante en la respuesta educativa a este alumnado: las NEE asociadas a la historia educativa y escolar desajustada o carencias en el entorno sociofamiliar nunca tuvieron, en la práctica, una respuesta organizada, ni se dispusieron los recursos ni las ayudas adecuadas para compensarlas. Salvo en 


\section{DIFICULTADES Y CONTRADICCIONES EN LA RESPUESTA EDUCATIVA
I 58 PARA LOS ALUMNOS CON NECESIDAD ESPECÍFICA DE APOYO EDUCATIVO (ACNEAE) ROBERTO RUIZ ANDRÉS}

centros educativos grandes, situados en núcleos de población importantes, en los cuales se implantaron los Programas de Educación Compensatoria, en el resto de los casos la Administración Educativa se limitaba a registrar a este alumnado en listados. La respuesta educativa queda reducida a las intervenciones que el profesorado ordinario (tutores, sobre todo) pudiera articular.

Queda así establecida una diferenciación importante entre un tipo y otro de necesidades: las que se asocian a condiciones personales (internas/estables) de discapacidad y aquellas cuyas causas se atribuyen al entorno familiar y/o sociocultural. Vemos en esta diferenciación cómo, incluso en pleno auge del «modelo de necesidades educativas especiales», vuelve a aflorar el viejo «modelo del déficit» tanto en la conceptualización de las NEE como en los modos de responder a las mismas, perpetuándose hasta nuestros días.

La estrategia para dar una respuesta educativa a todos los ACNEE era la adaptación curricular: adaptaciones curriculares significativas, adaptaciones curriculares no significativas y adaptaciones de acceso. Las primeras suponen una modificación importante de los elementos prescriptivos del currículo (objetivos, contenidos y criterios de evaluación). En principio, todos los ACNEE podían utilizar cualquiera de los tipos de adaptación si las necesitasen.

\subsection{Alumnos con necesidad especifica de apoyo educativo (ACNEAE)}

La LOE (2006) -y lo mantiene la LOMCE (2013)- introduce el término de alumnos con necesidad específica de apoyo educativo para referirse a todos aquellos alumnos que

[...] requieran una atención educativa diferente a la ordinaria, por presentar necesidades educativas especiales, por dificultades específicas de aprendizaje, TDAH, por sus altas capacidades intelectuales, por haberse incorporado tarde al sistema educativo, o por condiciones personales o de historia escolar... (art. 71.2).

En Castilla y León, la Instrucción de 24 de agosto de 2017 de la Dirección General de Equidad e Innovación Educativa y Formación del Profesorado concreta cinco grupos de Alumnos con Necesidad Específica de Apoyo Educativo (ACNEAE): Alumnado con Necesidades Educativas Especiales (ACNEE), Alumnado con Necesidades de Compensación Educativa (ANCE), Altas Capacidades Intelectuales, Dificultades de Aprendizaje y/o Bajo Rendimiento Académico y Trastorno por Déficit de Atención e Hiperactividad (TDAH).

Como vemos, dentro del gran grupo de todos los ACNEAE se diferencia el subgrupo de alumnos con necesidades educativas especiales (ACNEE) y se define como:

[...] aquel que requiera, por un periodo de su escolarización o a lo largo de toda ella, determinados apoyos y atenciones educativas específicas derivadas de discapacidad o trastornos graves de conducta (LOE, 2006: art. 73).

Queda así restringido el concepto de NEE, ya que estas solo pueden asociarse a discapacidad o trastornos graves de conducta y no a cualquier causa como antes; dicho de otro modo, ya no podemos hablar de ACNEE cuando sus necesidades educativas derivan de otros factores como familiares, socioculturales o dificultades específicas 


\section{DIFICULTADES Y CONTRADICCIONES EN LA RESPUESTA EDUCATIVA PARA LOS ALUMNOS CON NECESIDAD ESPECÍFICA DE APOYO EDUCATIVO (ACNEAE) ROBERTO RUIZ ANDRÉS}

de aprendizaje, entre otros. Esta nueva clasificación afecta a la respuesta educativa. Veamos algunas de las repercusiones que tiene sobre la práctica.

\section{Implicaciones para la respuesta educativa de la nueva clasificación de ACNEAE (LOE, 2006). Aspectos positivos y negativos}

Discutimos a continuación sobre algunos aspectos positivos y negativos que observamos derivados de esta nueva clasificación realizada a partir de la LOE (2006) de los alumnos que presentan dificultades mayores que sus compañeros para alcanzar el máximo desarrollo posible de sus capacidades personales y, en todo caso, los objetivos establecidos con carácter general para todo el alumnado.

\section{I. Aspectos positivos: recursos necesarios para todos los ACNEAE y menor número de alumnos en la denostada categoría de ACNEE}

En primer lugar, observamos un avance importante en el hecho de contemplar ahora como alumnado con necesidad específica de apoyo educativo a muchos alumnos para los que la normativa anterior, a pesar de considerarlos como ACNEE, no diseñaba ni desarrollaba en la práctica una respuesta educativa ajustada a sus necesidades. Nos referimos, como hemos apuntado anteriormente, a aquellos alumnos que presentando necesidades educativas especiales derivadas de carencias del entorno familiar, o de una historia escolar desajustada, o de dificultades específicas de aprendizaje, no llegaban a «entrar en el diagnóstico» con cierto grado de discapacidad y, a pesar de estar registrados como ACNEE por la Administración Educativa, nunca llegaron a recibir ni los recursos ni la respuesta adecuada que les brindaba la normativa en aquella época (Real Decreto 696/1995 sobre ordenación de la educación de los alumnos con necesidades educativas especiales y la Orden de i4 de febrero de 1996 sobre evaluación psicopedagógica, ambas disposiciones hoy derogadas). Podemos exceptuar algunos casos de alumnos que se incorporaban al Programa de Educación Compensatoria y que sí recibían apoyo educativo «extra» por parte de un maestro. En la actualidad, con la normativa vigente, y con la nueva clasificación que venimos comentando, para todos los alumnos incorporados al grupo de ACNEAE, ya queda contemplada la obligación por parte de las administraciones educativas de disponer y asegurar los recursos necesarios y adecuados para compensar sus dificultades y para que todos alcancen los objetivos del currículo. Así se recoge en la LOMCE (20I3): "Corresponde a las Administraciones educativas asegurar los recursos necesarios para que los alumnos y alumnas que requieran una atención educativa diferente a la ordinaria [...] puedan alcanzar el máximo desarrollo posible de sus capacidades personales y, en todo caso, los objetivos establecidos con carácter general para todo el alumnado (art. 7I)». Ello, desde nuestro punto de vista, no deja de ser un aspecto positivo con la correspondiente repercusión sobre la práctica.

En segundo lugar, y desde un punto de vista más subjetivo, otro aspecto positivo que se ha podido derivar de esta nueva clasificación es que se reduce a un menor número el alumnado etiquetado y clasificado con la denostada categoría de ACNEE por el hecho de sacar de este grupo a alumnos de Compensación Educativa (ANCE), a alumnos 


\section{DIFICULTADES Y CONTRADICCIONES EN LA RESPUESTA EDUCATIVA I 60 PARA LOS ALUMNOS CON NECESIDAD ESPECÍFICA DE APOYO EDUCATIVO (ACNEAE) ROBERTO RUIZ ANDRÉS}

de Altas Capacidades y a Alumnos con Dificultades Específicas de Aprendizaje y/o Bajo Rendimiento Académico. Pensamos que de esta forma se da una imagen de mayor normalización de las dificultades en la escuela, lo cual puede contribuir a su vez a generar la idea de que todos los alumnos tienen necesidades educativas, muchos tienen necesidades específicas de apoyo y unos pocos presentan necesidades especiales; por otro lado, pueden crearse mayores expectativas sobre muchos ACNEAE al no quedar ya incorporados al grupo de ACNEE. Son cuestiones que requieren un debate con mayor profundidad, no es objeto de discusión en este documento.

\subsection{Aspectos negativos. Alteración y pérdida del carácter relativo, interactivo y continuo de las NEE}

Como ya hemos apuntado anteriormente, desde el origen mismo del concepto de NEES ya se produjo en la práctica una gran diferenciación entre las NEE asociadas a discapacidad, para las cuales sí se contemplaba una respuesta educativa ajustada a las mismas (recursos de maestros de Pedagogía Terapéutica-PT-, Audición y Lenguaje -AL-, Ayudante Técnico Educativo-ATE-...) y aquellas otras NEE que se derivaban de otras causas (historia escolar desajustada, ambientes familiares no favorecedores o dificultades específicas de aprendizaje), para las que no se contemplaban, normalmente, ayudas «extra». La nueva clasificación de ACNEAE viene a perpetuar esta diferenciación mediante la asociación de las NEE exclusivamente a condiciones de discapacidad o trastornos graves de conducta (condiciones personales más estables y permanentes) $y$, por ende, a consolidar la idea de que las NEE no pueden derivarse de cualquier otra causa. Desde nuestro punto de vista esto constituye un error, puesto que nos encontramos en la práctica con alumnos con necesidades educativas asociadas a factores familiares, sociales y culturales, o a dificultades específicas de aprendizaje que pueden ser tan especiales y requerir respuestas y adaptaciones tan específicas como aquellas que se derivan de discapacidad o trastornos graves de conducta. Por ejemplo, actualmente es frecuente encontrar en las aulas alumnado procedente de ambientes familiares muy desestructurados; entonces nos preguntamos: ¿se puede afirmar que dichas necesidades no son especiales?, ¿se puede afirmar que el tipo y grado de ayuda que precisan estos alumnos no es especial?, ¿por qué tienen que ser más especiales las necesidades educativas derivadas de una discapacidad intelectual leve, por ejemplo, que esas otras derivadas de ambientes familiares muy desestructurados o de un trastorno específico de aprendizaje?

Una idea esencial en el concepto mismo de necesidades educativas especiales es el carácter relativo, interactivo y continuo de las mismas. Así expresa Blanco el carácter interactivo y relativo inherente al concepto mismo de NEE y que, a nuestro juicio, se está perdiendo o alterando actualmente:

[...] la «dimensión real» de las dificultades de aprendizaje de los alumnos tiene un carácter fundamentalmente interactivo: dependen tanto de las características personales del alumno como de las características del entorno educativo en el que éste se desenvuelve y la respuesta educativa que se le ofrece. Pensar que las dificultades están sólo en el alumno, por ejemplo - «es deficiente mental y por eso tiene dificultades para aprender»conlleva, por lo general, un bajo nivel de expectativas respecto de las posibilidades de 

ROBERTO RUIZ ANDRÉS

estos alumnos y un menor compromiso por parte de la escuela para dar respuesta a sus dificultades. [...]

Por otro lado, partir de un carácter interactivo de las dificultades de aprendizaje nos remite a la idea de relatividad de las necesidades educativas especiales, ya que éstas serán diferentes en función de las características y respuesta educativa que se ofrece en cada contexto educativo (Blanco, 1996: 23).

Entendemos que con esa diferenciación de necesidades educativas comentada se contribuye a desvirtuar el concepto mismo de NEE alterando las características esenciales que lo configuran: pérdida del carácter relativo, interactivo y continuo de dichas NEE. Al asociarse ahora las NEE solo a discapacidad o trastornos graves de conducta (causas internas y estables) y no como antes a cualquier causa, se vuelve a poner el acento, con más énfasis si cabe, solo en las dificultades del alumno, en el tipo y grado de discapacidad, debido a que las necesidades se vinculan y atribuyen a causas internas y estables, sin considerar que, en realidad, esas necesidades son relativas y siempre están en interacción con el entorno educativo en el que se presentan y que en gran medida van a depender de las transformaciones que de ese mismo entorno seamos capaces de articular para que todos puedan participar, aprender y tener éxito.

Observamos, además, que con esa nueva clasificación que establece la LOE (2006) al separar firmemente las NEE y los alumnos que las presentan (que solo pueden asociarse a discapacidad o trastornos graves de conducta) del resto de las necesidades educativas del resto de los ACNEAE no solo se ha perdido la consideración del carácter relativo e interactivo de las necesidades educativas (se sigue pensando que el déficit está en el alumno), sino que también se ha producido una alteración de esa idea esencial de continuum tanto en el concepto mismo de NEE, como en el de la adaptación curricular que debe responder a ellas. Es decir, que paralelamente a esa categorización aparece también una gran diferenciación de medidas, recursos y ayudas. Nos remitimos al hecho concreto de que se reserven las adaptaciones curriculares significativas exclusivamente para ACNEE, cuando en realidad otros grupos de ACNEAE las necesitan para progresar y avanzar hacia el logro de los objetivos comunes de la educación, pues su nivel de competencia curricular frecuentemente se aparta significativamente del currículo ordinario, quedando así la respuesta educativa para este alumnado poco definida, poco ajustada a las necesidades reales que presenta y diluida en unas medidas generales, poco eficaces y, a menudo, incongruentes.

\section{Un continuo de necesidades exige un continuo de adaptación curricular}

Junto al concepto mismo de NEE, surgía ya en los años 9o (LOGSE, I99o) la idea de que para que los alumnos accedieran y realizaran los aprendizajes establecidos en un currículo común, comprensivo y alcanzaran los mismos fines de la educación, era preciso utilizar ayudas y realizar adaptaciones oportunas de dicho currículo. Aparecía entonces una estrategia genuina que salvaría la dificultad de hacer compatible, y de articular equilibradamente, un currículo común para todos, con la diversidad del alumnado: la adaptación curricular.

Una característica básica de las adaptaciones curriculares es que forman un continuo. Las NEE que pueden presentar los alumnos van, en realidad, desde las más leves 


\section{DIFICULTADES Y CONTRADICCIONES EN LA RESPUESTA EDUCATIVA I 62 PARA LOS ALUMNOS CON NECESIDAD ESPECÍFICA DE APOYO EDUCATIVO (ACNEAE) ROBERTO RUIZ ANDRÉS}

y transitorias hasta las más graves y permanentes, aparecen formando un continuo, como continua también debe ser la ayuda o adaptación que se necesita para compensarlas..., así se afirmaba en el Libro Blanco (1989) y en la propia Ley Orgánica (LOGSE, 1990). Del mismo modo lo recoge Rosa Blanco (1996) [...] «Las adaptaciones curriculares precisas para compensar las dificultades de aprendizaje de los alumnos constituyen un continuo que va desde ajustes poco significativos del planteamiento educativo común a modificaciones más significativas, y desde ajustes temporales a modificaciones más permanentes» (p. 27). Dichas modificaciones pueden ser: adaptaciones de acceso al currículo, adaptaciones curriculares no significativas y adaptaciones curriculares significativas, dependiendo del grado de modificación del currículo y de a qué elementos del mismo afecten.

En Castilla y León, tanto en la Orden EDU/II52/20Io de 3 de agosto, como en la Orden EDU/362/2015 de 4 de mayo, se establece la división entre: a) Medidas generales $u$ ordinarias de atención a la diversidad. Destinadas a todos los alumnos y se corresponderían con las adaptaciones curriculares no significativas. Afectan «[...] a la metodología, a la organización, a la adecuación de las actividades, a la temporalización y a la adaptación de las técnicas, tiempos e instrumentos de evaluación [...]. En todo caso, estas adaptaciones tomarán como referente los criterios de evaluación establecidos con carácter general en las correspondientes programaciones didácticas» (Orden EDU/II52/20IO, art. 6). b) Medidas especificas (según la Orden EDU/II52/20IO) o especializadas y extraordinarias (según la Orden EDU/362/2015) de atención a la diversidad. Son aquellas medidas que pueden implicar, entre otras, la modificación significativa de los elementos del currículo para su adecuación a las necesidades de los alumnos. Entre las especializadas aparecen:

Adaptaciones de accesibilidad al currículo para el alumnado con necesidades educativas especiales, así como los recursos de apoyo que les permitan acceder al currículo.

Adaptaciones curriculares significativas de los elementos del currículo dirigidas al alumnado con necesidades educativas especiales. Se realizarán buscando el máximo desarrollo posible de las competencias; la evaluación continua y la promoción tomarán como referencia los elementos fijados en ellas (Orden EDU/362/2015: art. 26.4).

Las adaptaciones significativas suponen la modificación sustancial, generalmente por eliminación, de alguno de los elementos prescriptivos del currículo como objetivos de la etapa, contenidos, criterios de evaluación o estándares de aprendizaje evaluables. Normalmente hablamos de adaptación significativa en una determinada materia para un alumno cuando se realiza una modificación correspondiente a dos o más cursos de desfase con respecto a la programación de aula. En Castilla y León, la Resolución de 17 de agosto de 2009, además, lo resalta de manera contundente:

Las adaptaciones curriculares significativas se elaborarán exclusivamente para el alumnado con necesidades educativas especiales que se encuentre en alguna de las siguientes situaciones:

a) Que presente un desfase importante en su desarrollo personal [...].

b) Que presente un desfase curricular de un ciclo en el segundo y tercer ciclo de educación primaria o de dos cursos en educación secundaria obligatoria, entre su nivel de competencia curricular y el curso en el que efectivamente se encuentre escolarizado. 

ROBERTO RUIZ ANDRÉS

De la misma forma, el Real Decreto Iro5/20I4 indica que «Las Administraciones educativas [...] establecerán los procedimientos oportunos cuando sea necesario realizar adaptaciones significativas de los elementos del currículo, a fin de atender al alumnado con necesidades educativas especiales que las precise...» (art. 9). Observamos cómo en toda la normativa las adaptaciones curriculares significativas se reservan exclusivamente para ACNEE. Sin embargo, muchos otros alumnos, que no presentan NEE, tienen un desfase de dos o más cursos en su nivel de competencia curricular.

En síntesis, la gran distinción que se establece entre las NEE y aquellas otras necesidades educativas derivadas de dificultades específicas de aprendizaje, de un ambiente familiar desfavorecido o de una historia escolar desajustada lleva a tender una enorme barrera que separa las adaptaciones curriculares significativas, destinadas exclusivamente a ACNEE (por normativa) las cuales toman como referentes para la evaluación los elementos fijados en ellas, y las adaptaciones curriculares no significativas destinadas a todos los alumnos, también para ACNEAE que no sean ACNEE las cuales toman los referentes para la evaluación en el currículo ordinario, aunque presenten un desfase curricular significativo. Ello supone ciertos dilemas y dificultades a la hora de diseñar y evaluar la propuesta educativa para este alumnado.

\section{Dilemas en el diseño y en la evaluación de la propuesta curricular individual}

Es mayúscula la incongruencia con la que nos encontramos en la práctica educativa a la hora de diseñar, desarrollar y evaluar la propuesta educativa adaptada para la mayoría de ACNEAE que no pertenecen al grupo de ACNEE (alumnado al que nos venimos refiriendo a lo largo de todo el documento: Alumnos de Compensación Educativa -ANCE-, Alumnos con Dificultades Específicas de Aprendizaje y/o Bajo Rendimiento Académico y entre ellos sobre todo los alumnos con Capacidad Intelectual Límite, o para algunos alumnos con Trastorno de Déficit de Atención con Hiperactividad). Este alumnado puede presentar, y de hecho presentan con muchísima frecuencia, un desfase en la competencia curricular de dos o más cursos con respecto al de su grupo de referencia en la mayoría de las materias. Encontramos grandes dilemas y dificultades tanto a la hora de diseñar una propuesta curricular individualizada como en el momento de afrontar la evaluación de los aprendizajes, sobre todo en las materias de Lengua y Matemáticas ya que son las asignaturas en las que recibe apoyo directo, normalmente fuera del aula ordinaria.

\section{I. A la hora de diseñar una propuesta curricular individualizada}

Como dijimos al presentar el documento, normalmente la respuesta educativa que se le ofrece a este alumnado en los centros de educación secundaria se limita a un apoyo individualizado en las materias de Lengua y de Matemáticas. Habitualmente lo reciben en las aulas de apoyo (Pedagogía Terapéutica y de Educación Compensatoria). Se diseña para estas materias una propuesta curricular individualizada que parte de su NCC, es decir, con una distancia curricular significativa de al menos dos cursos con respecto a la Programación de Aula. Dicha propuesta supone la modificación (eliminación y priorización) de los elementos básicos del currículo (objetivos, contenidos 


\section{DIFICULTADES Y CONTRADICCIONES EN LA RESPUESTA EDUCATIVA I 64 PARA LOS ALUMNOS CON NECESIDAD ESPECÍFICA DE APOYO EDUCATIVO (ACNEAE) ROBERTO RUIZ ANDRÉS}

y criterios de evaluación) de manera importante, «significativa». No podemos hablar de adaptación curricular significativa (ACS), pero realmente esta propuesta lo es. Ante esto nos preguntamos: ¿se deben elaborar Acs significativas en Lengua y Matemáticas cuando existe un desfase curricular de más de dos cursos en estas asignaturas? Esta pregunta sugiere dos tipos de respuestas. Respuesta I: no, las adaptaciones curriculares significativas se elaborarán (por normativa) exclusivamente para el alumnado con necesidades educativas especiales y este alumnado no entra en ese grupo. Respuesta 2: sí, pues, por principio psicopedagógico fundamental, es preciso partir del nivel de competencia curricular del alumno y diseñar una propuesta curricular adaptada para que, a partir de lo que sabe, pueda aprender y progresar; así, si el Ncc del alumno se sitúa a una distancia de dos o más cursos con respecto al currículo ordinario, deberemos realizar una ACs (por definición propia de ACs).

La normativa, como hemos visto, por un lado, nos dice que no podemos realizar ACs para este alumnado, pues se reservan exclusivamente para ACNEE, al mismo tiempo nos dice que debemos realizar adaptaciones significativas cuando exista un desfase de dos cursos o más, pero solo si son ACNEE. Nos preguntamos entonces cómo podemos articular en la práctica el concepto de adaptación curricular significativa con el hecho de que esta medida de atención a la diversidad se reserve exclusivamente para ACNEE.

\subsection{A la hora de afrontar la evaluación de los aprendizajes}

La evaluación constituye uno de los elementos más importantes del proceso de E/A, es un aspecto de ese mismo proceso amplio, delicado y complejo, pensamos por ello que no debemos caer en simplismos absurdos o en planteamientos burdos como el que prescribe la normativa actual y que venimos denunciando en este documento. A través de esta cita queremos expresar la importancia que debiéramos dar a la evaluación y las implicaciones que puede tener cuando hablamos de alumnos vulnerables.

La evaluación es un tema constante de preocupación de los docentes, de las autoridades escolares y de los investigadores de la educación. Esto indica de por sí que se trata de un punto sensible, muy cargado afectiva e ideológicamente, porque a través de la evaluación se concretan las desigualdades y, por tanto, las contradicciones, entre la utopía pedagógica de «una escuela para todos» y la realidad de las diferencias (Perrenoud, I990: 29-30. Citado por Ruiz Andrés, 2016: III).

Toda la normativa consultada contempla el proceso de evaluación como un elemento esencial del proceso mismo de enseñanza-aprendizaje. Por seleccionar una de esas normativas, el $\mathrm{RD} / \mathrm{IIO}_{\mathrm{O}} / 20 \mathrm{I} 4$, el artículo 20 recoge que:

La evaluación del proceso de aprendizaje del alumnado de la Educación Secundaria Obligatoria será continua, formativa e integradora.

La evaluación de los aprendizajes de los alumnos y alumnas tendrá un carácter formativo y será un instrumento para la mejora tanto de los procesos de enseñanza como de los procesos de aprendizaje.

Las Administraciones educativas garantizarán el derecho de los alumnos a una evaluación objetiva y a que su dedicación, esfuerzo y rendimiento sean valorados y reconocidos con objetividad, para lo que establecerán los oportunos procedimientos. 

ROBERTO RUIZ ANDRÉS

A la hora de evaluar a este alumnado nos encontramos con el siguiente dilema: debemos enseñar, y el alumno debe aprender, los objetivos/contenidos establecidos en su propuesta curricular individual (normalmente con una distancia de dos cursos en el NCC), pero a la hora de evaluar debemos tomar los referentes de la Programación de Aula. Ante esto, nos preguntamos, entre otras cuestiones: ¿cómo podemos trabajar y enseñar objetivos y contenidos de un determinado nivel fijado en el diseño de la Propuesta Curricular Individualizada o Plan de Apoyo Individualizado y luego evaluar los aprendizajes del alumno utilizando los referentes correspondientes a dos cursos (por lo menos) por encima de ese nivel establecido?, ¿qué tipo de planteamiento educativo es este que trabaja y enseña unos objetivos/contenidos y evalúa otros?, ¿dónde queda el sentido de la evaluación si la desvinculamos del proceso de E/A?, etc.

El resultado más evidente de este planteamiento es que el alumno ya, a priori, está abocado al fracaso y a la evaluación/calificación negativa con las consecuencias que ello acarrea. De este modo, la evaluación del alumnado más vulnerable del centro es un elemento de discriminación y de exclusión. Así la evaluación se convierte en un instrumento con el que «[...] la escuela transforma la diversidad en diferencia. Las pequeñas y naturales diferencias como podrían ser la diferencia de capacidades o el estilo de aprendizaje, la escuela las va transformando en diferencias cada vez mayores» (Ruiz Andrés, 2016: 135).

\section{Incertidumbres y controversias ante los dilemas planteados}

Estas incongruencias en la propia normativa, que derivan en los dilemas que hemos comentado, vienen generando dudas, incertidumbres y desacuerdos entre los profesionales, y han llevado a los diferentes centros de educación secundaria a adoptar criterios y prácticas muy dispares a la hora de planificar la propuesta curricular individual y, sobre todo, la evaluación en las materias de Lengua y Matemáticas para este alumnado al que nos venimos refiriendo.

Para la evaluación, desde el aula de apoyo (de pedagogía terapéutica y educación compensatoria) se elabora un informe individual cualitativo trimestral en el que se especifican los objetivos/contenidos trabajados, los logros alcanzados, actitud del alumno, dificultades, etc. Este informe se entrega al alumno junto con el boletín de las calificaciones oficiales. Donde existe controversia y disparidad de actuaciones es a la hora de establecer la calificación oficial mediante el programa informático (en nuestro centro IES Fácil) que configura el boletín de calificaciones que el alumno llevará a casa y en el que por defecto aparece una única observación ME (Medidas Educativas). Podemos distinguir dos opiniones o posiciones encontradas. Posición primera: por un lado, se opina que, independientemente de que el alumno haya logrado sobradamente los aprendizajes establecidos en su Plan de Apoyo Individual, mientras no alcance los objetivos/contenidos de la Programación de Aula debe estar suspenso en las calificaciones oficiales de cada trimestre, y si al finalizar el curso hubiera llegado a alcanzar los mínimos de la Programación de Aula, podría obtener calificación positiva. Entendemos que difícilmente, partiendo de un desfase de dos cursos o más, este alumnado con dificultades de aprendizaje llegue al final de curso a alcanzar los aprendizajes establecidos en el nivel de la Programación de Aula. El argumento principal que ofrecen los defensores de esta postura es que, de este modo, no se induce a 


\section{DIFICULTADES Y CONTRADICCIONES EN LA RESPUESTA EDUCATIVA
I 66 PARA LOS ALUMNOS CON NECESIDAD ESPECÍFICA DE APOYO EDUCATIVO (ACNEAE) ROBERTO RUIZ ANDRÉS}

engaños o malentendidos sobre el nivel real de competencia curricular del alumno. Posición segunda: por otro lado, se opina (somos partidarios de esta opinión) que mientras este alumno haya alcanzado sobradamente los objetivos/contenidos planteados en su propuesta curricular individual (desarrollada mayormente en el aula de apoyo) con esfuerzo y trabajo al nivel de sus posibilidades, con una buena actitud y disposición, etc., este alumno debería obtener una calificación positiva de sus aprendizajes y quedar así reflejada, con las oportunas aclaraciones y observaciones, en el boletín oficial de calificaciones. De este modo se evalúa lo que ha logrado el alumno en relación con lo que se ha enseñado en el plan individual, considerando así la evaluación como parte importantísima dentro del proceso mismo de E/A, con un alto valor formativo que le es propio, como indica toda la teoría al respecto.

Algunas de las razones que nos hacen posicionarnos en esta segunda opción ya las hemos mencionado: no se puede separar la evaluación del proceso de E/A. En este sentido, si a lo largo del trimestre o del curso trabajamos objetivos y enseñamos contenidos correspondientes a un determinado nivel, ¿cómo vamos a manejar los criterios de evaluación y estándares de aprendizaje correspondientes a un nivel dos cursos por encima a la hora de abordar la evaluación?, ¿cómo podemos evaluar y calificar incluso objetivos/contenidos que no se han trabajado con el alumno?; así, este alumnado siempre va a estar evaluado negativamente, haga lo que haga en la clase de apoyo, trabaje lo que trabaje y logre lo que logre. A modo de reflexión, vamos a ofrecer algunas razones más para rechazar la primera postura a través de una serie de preguntas: ¿con qué ética profesional podemos exigir al alumno esfuerzo y a los padres que se impliquen en el proceso educativo de su hijo sabiendo que las metas curriculares están tan alejadas que, al menos por ese curso, no se van a lograr?, ¿cómo informar al alumno y a los padres de que, a pesar del esfuerzo o interés que ponga en el trabajo diario, siempre va a suspender en cada sesión de evaluación?, ¿podemos imaginar cómo puede afectar esta forma de evaluar a aspectos como la autoestima y motivación del alumno, a las expectativas de los padres y a las del profesor, entre otros? (aspectos normalmente ya fuertemente afectados por la historia escolar de este alumnado), ¿podemos valorar, siguiendo la literatura especializada, la incidencia de estas cuestiones sobre los aprendizajes? En fin, ¿cómo podemos presentar al propio alumno, a los padres, a nosotros mismos como docentes y a la Administración Educativa un planteamiento de respuesta educativa como este que enseña unos contenidos/objetivos, pero evalúa otros?

Reclamamos de manera urgente una modificación y adecuación de las diferentes normativas que ayuden a resolver los dilemas planteados y a solventar las dudas, la incertidumbre y las controversias que se generan en los centros educativos sobre el asunto. Mientras llegan estas modificaciones proponemos seguir las pautas descritas en la posición segunda de este apartado. En la provincia de Burgos estamos trabajando en la elaboración de una encuesta a los profesionales de los diferentes centros de educación secundaria para conocer los diversos modos en los que se llevan a cabo el diseño de la respuesta educativa y la evaluación de este alumnado a fin de poder consensuar criterios comunes de actuación.

\section{Conclusiones}

La respuesta educativa que se ofrece en los centros educativos de educación secundaria a determinados ACNEAE es claramente mejorable y exige trasformaciones 


\section{DIFICULTADES Y CONTRADICCIONES EN LA RESPUESTA EDUCATIVA PARA LOS ALUMNOS CON NECESIDAD ESPECÍFICA DE APOYO EDUCATIVO (ACNEAE) I 67 ROBERTO RUIZ ANDRÉS}

estructurales y de calado en el Sistema que supongan un cambio profundo de culturas, políticas y prácticas educativas. Es necesaria una revisión profunda sobre dicha respuesta para avanzar en consonancia con las premisas del modelo de escuela inclusiva que pretendemos.

Existe una enorme brecha entre, por un lado, los preceptos de la normativa educativa y lo que también sugieren la literatura y la investigación sobre la atención a la diversidad en la escuela y, por otro, lo que realmente viene ocurriendo en las aulas y en los centros de educación secundaria. Llevamos casi tres décadas intentando superar el modelo del déficit y situarnos en el modelo de necesidades educativas especiales de cara a prestar la atención educativa a los alumnos que presentan mayores dificultades que el resto de sus compañeros para acceder al currículo común y adquirir los objetivos, y no lo hemos conseguido plenamente. Pensamos que realmente estamos atrapados aún en un modelo de atención a la diversidad trasnochado: el modelo del déficit, que muestra las actitudes y la conceptualización que aún hoy existe sobre las dificultades de aprendizaje, sobre las NEE y sobre los alumnos que las presentan. Esa misma conceptualización, estas ideas y actitudes que imperan en la práctica educativa actualmente están presidiendo, gobernando y dirigiendo erróneamente las tareas de identificación y determinación de dichas necesidades y, por consiguiente, de organización de la respuesta educativa que se necesita para compensarlas. Por lo tanto, no habiendo asumido, aún siquiera plenamente en la práctica, el modelo de necesidades educativas especiales propuesto en los años 90 (como se analiza detenidamente en Ruiz Andrés [20I6]), cómo vamos a pretender superar este modelo y dar el salto al modelo de la identificación y eliminación de barreras para la presencia, la participación y el aprendizaje de todos, es decir, el salto al modelo inclusivo.

Con el presente documento hemos pretendido dejar patente que, al menos en los centros de educación secundaria, estamos desarrollando políticas y prácticas concretas, orientadas por determinadas actitudes y modos de entender la diversidad, que hacen que la respuesta educativa que ofrecemos a determinados alumnos no sea la más adecuada, ni la más justa ni la de mayor calidad y equidad y que están generando desigualdad, fracaso y exclusión. Consideramos que debemos seguir reflexionando sobre qué actitudes, ideas y creencias tenemos acerca de la diversidad en el centro educativo; sobre qué políticas organizativas hemos instaurado y normalizado, y sobre qué prácticas concretas venimos desarrollando como docentes para que podamos encontrar, entre todos, ideas y estrategias para ir tomando el camino hacia un modelo de respuesta educativa más inclusivo y de mayor calidad. A la postre se trata de encontrar la forma de lograr que el centro educativo haga valer, por justicia, el derecho a la inclusión y asuma plenamente la responsabilidad de ofrecer una educación de calidad para todos y con todos.

\section{Bibliografía}

Acuerdo 29/20I7, de is de junio, de la Junta de Castilla y León, por el que se aprueba el II Plan de Atención a la Diversidad en la Educación de Castilla y León 2017-2022.

Ainscow, M. y Miles, S. (2008). Por una educación para todos que sea inclusiva: ¿Hacia dónde vamos ahora? Perspectivas, 38(I), 17-44.

Arnárz, P. (2003). Educación inclusiva. Una escuela para todos. Málaga: Aljibe. 
I 68 DIFICULTADES Y CONTRADICCIONES EN LA RESPUESTA EDUCATIVA PARA LOS ALUMNOS CON NECESIDAD ESPECÍFICA DE APOYO EDUCATIVO (ACNEAE) ROBERTO RUIZ ANDRÉS

Blanco, R. (coord.). (1996). Alumnos con necesidades educativas especiales y adaptaciones curriculares. Madrid: MEC-Centro Nacional de Recursos para la Educación Especial.

Bоотн, T. y Ainscow, M. (2002). Guía para la evaluación y mejora de la educación inclusiva. Desarrollando el aprendizaje y la participación en las escuelas. Madrid: Consorcio Universitario para la Educación Inclusiva.

CNREE (1992). Alumnos con necesidades educativas especiales y adaptaciones curriculares. Madrid: MEC.

Echeita, G. y Calderón, I. (20I4). Obstáculos a la inclusión: cuestionando concepciones y prácticas sobre la evaluación psicopedagógica. Ámbitos de Psicopedagogía y Orientación, n. ${ }^{\circ} 4 I\left(2 .^{a}\right.$ época).

Echeita, G. y Domínguez Gutiérrez, A. B. (20II). Educación Inclusiva. Argumento, Caminos, Encrucijadas. Aula, Revista de Pedagogía de la Universidad de Salamanca, I7, 23-35.

Instrucción de 24 de agosto de 2017 de la Dirección General de Innovación y Equidad Educativa por la que se modifica la Instrucción de 9 julio de 2015 de la Dirección General de Innovación Educativa y Formación del Profesorado, por la que se establece el procedimiento de recogida y tratamiento de los datos relativos al alumnado con necesidad específica de apoyo educativo escolarizado en centros docentes de Castilla y León.

Ley Orgánica i/1990, de 3 de octubre, de Ordenación General del Sistema Educativo. BOE núm. 238 , de 4 de octubre de 1990.

Ley Orgánica 2/2006, de 3 de mayo, de Educación. $B O E$ núm. Io6, de 4 de mayo de 2006.

Ley Orgánica 8/2013, de 9 de diciembre, para la mejora de la calidad educativa. $B O E$ núm. 295, de ro de diciembre de 2013 .

MEC (1989). Libro Blanco para la reforma del Sistema Educativo. Madrid: MEc.

MEC (1994). La Educación Especial en el marco de la LOGSE. Madrid: Centro de Publicaciones del MEc.

Orden EDU/II52/20IO, de 3 de agosto, por la que se regula la respuesta educativa al alumnado con necesidad específica de apoyo educativo escolarizado en el segundo ciclo de Educación Infantil, Educación Primaria, Educación Secundaria Obligatoria, Bachillerato y Enseñanzas de Educación Especial, en los centros docentes de la Comunidad de Castilla y León. BOCyL núm. 156 , de 13 de agosto de 2010.

Orden EDU/362/20I5, de 4 de mayo, por la que se establece el currículo y se regula la implantación, evaluación y desarrollo de la educación secundaria obligatoria en la Comunidad de Castilla y León. BOCyL núm. 86, de 8 de mayo de 2015.

Real Decreto 696/1995, de 28 de abril, de ordenación de la educación de los alumnos con necesidades educativas especiales. $B O E$ núm. I3I, de 2 de junio de 1995 .

Real Decreto IIo5/20I4, de 26 de diciembre, por el que se establece el currículo básico de la Educación Secundaria Obligatoria y del Bachillerato. $B O E$ núm. 3, de 3 enero de 2015.

Resolución de 17 de agosto de 2009, de la Dirección General de Planificación, Ordenación e Inspección Educativa, por la que se regula el diseño, aplicación, seguimiento y evaluación de las adaptaciones curriculares significativas para el alumnado con necesidades educativas especiales escolarizado en el segundo ciclo de educación infantil, educación primaria y educación secundaria obligatoria en los centros docentes de la Comunidad de Castilla y León. BOCYL núm. 162, de 26 de agosto de 2009.

Ruiz Andrés, R. (2016). Medidas de atención a la diversidad: una experiencia de aula alternativa de adaptación e intervención educativa en Burgos para alumnos en riesgo de fracaso y abandono escolar. Tesis doctoral inédita. Burgos: Universidad de Burgos. 